\title{
Serum 25-hydroxyvitamin D is negatively associated with severe periodontitis: a cross-sectional study
}

Fangjing Zhou ${ }^{1,3}$, Ning Ma ${ }^{1}$, Ruiting Su${ }^{1}$, Xiaoyu He ${ }^{1}$, Xiaona Wang ${ }^{3}$, Yang Zhou ${ }^{2}$ and Jing Shi ${ }^{3 *}$

\begin{abstract}
Background: Periodontitis can lead to the destruction of periodontium and adversely influence the overall health, wellbeing, and quality of life. However, studies on the relationship between severe periodontitis and serum 25 -hydroxyvitamin $\mathrm{D}[25(\mathrm{OH}) \mathrm{D}]$ are limited. This study is designed to explore the relationship between $25(\mathrm{OH}) \mathrm{D}$ and severe periodontitis.

Methods: A cross-section study of 2928 participants enrolled from the National Health and Nutrition Examination Survey (NHANES) from 2013 to 2014 was conducted. The periodontal examination was performed using a total oral periodontal examination program, and probe measurements were collected at six sites per tooth in NHANES. Severe periodontitis was characterized as: $\geq 2$ interproximal sites with attachment loss (AL) $\geq 6 \mathrm{~mm}$ (not on the same tooth) and $\geq 1$ interproximal site with probing depth $(P D) \geq 5 \mathrm{~mm}$. Severe periodontitis and serum $25(\mathrm{OH}) \mathrm{D}$ were the dependent and independent variables, respectively. Univariate, multivariate, and subgroup analyses were performed to explore the relationship between severe periodontitis and serum 25(OH)D.
\end{abstract}

Results: Among the 2928 participants, the average age of the population was $50 \pm 13.71$ years old, with 1425 (48.67\%) males, 316 (10.79\%) exhibited severe periodontitis. Serum 25(OH)D showed a significantly negative association with severe periodontitis after adjusting all variables (OR 0.75, 95\% Cl 0.63-0.89). In addition, severe periodontitis has a nonlinear relationship with serum $25(\mathrm{OH}) \mathrm{D}$, whoes inflection point was $102(\mathrm{nmol} / \mathrm{L})$. On the left side of the inflection point $(25(\mathrm{OH}) \mathrm{D} \leq 102 \mathrm{nmol} / \mathrm{L})$, the effect size was 0.98 and $95 \% \mathrm{Cl}$ was $0.98-0.99(25(\mathrm{OH}) \mathrm{D}$ per $1 \mathrm{nmol} / \mathrm{L}$ increments). On the right side of the inflection point $(25(\mathrm{OH}) \mathrm{D}>102 \mathrm{nmol} / \mathrm{L})$, the effect size was 0.99 and $95 \% \mathrm{Cl}$ was 0.98-1.01. The subgroup analysis showed pronounced changes in non-Hispanic white, alcohol consumption, diabetes, and health insurance.

Conclusion: Serum $25(\mathrm{OH}) \mathrm{D}$ in relation to severe periodontitis is nonlinear in our study.When serum $25(\mathrm{OH}) \mathrm{D}$ is less than $102 \mathrm{nmol} / \mathrm{L}$, serum $25(\mathrm{OH}) \mathrm{D}$ is negatively associated with severe periodontitis.

Keywords: 25 -hydroxyvitamin D, Vitamin D, Periodontitis, Periodontal disease, NHANES

*Correspondence: crystalshi@163.com

${ }^{3}$ Department of Oral Medicine, The Fifth Clinical Medical College

of Shanxi Medical University, 22 Shuangtasi Street, Taiyuan 030012, Shanxi

Province, China

Full list of author information is available at the end of the article

\begin{abstract}
Background
Periodontitis is the sixth most prevalent disease globally with significant socioeconomic and systemic repercussions and is one of the most common chronic inflammatory diseases in adults [1]. An estimated $42 \%$ of the United States (U.S.) adults aged 30 years and over suffer from periodontal disease [2]. Periodontitis can lead to the
\end{abstract}


destruction of periodontium and adversely influence the overall health, wellbeing, and quality of life [3, 4].

Recent studies have reported an increasing relationship between periodontitis and diabetes, cardiovascular disease, inflammatory bowel disease, sleep disorders, chronic obstructive pulmonary disease, and other systematic diseases [5-7]. Periodontitis is a chronic inflammation disease caused by oral bacterial infection [8]. The body's immune response to the infection causes damage to the tissue. Vitamin D is essential to the general health, particularly in children, pregnancy, certain forms of cancer, and prevention of infection $[9,10]$. Moreover, studies indicated that serum Vitamin D deficiency is related to the severity and progression of periodontal disease $[5,11$, 12]. Periodontitis is a multifactorial disease that requires immune and complex infectious interactions [13].

In epidemiological studies, vitamin D supplement was inversely correlation with the incidence of periodontitis [14]. Studies have shown that $25(\mathrm{OH}) \mathrm{D}$ status could alter the risk for periodontitis by regulating the host's immune response to infection or by preventing alveolar bone loss [8]. Vitamin D is the general term for vitamin D2 and vitamin D3. The former is produced by ultraviolet radiation of yeast ergosterol, while the latter is produced by the ultraviolet radiation of 7-dehydrocholesterol in lanolin, has the biological activity of gallbladder calcium, and is synthesized in human skin [5]. A series of literatures have shown that 1,25-dihydroxyvitamin D3 could inhibit cytokine production and antigen-induced $\mathrm{T}$ cell proliferation [15]. However, there is limited clinical studies on the anti-inflammatory effects. Furthermore, Luo et al. found that the second highest level of vitamin D supplement have a lower severity of periodontitis compared to the highest level of vitamin D supplement [14]. It suggested that the correlation between periodontitis and vitamin D may be nonlinear. Recently, evidence indicated that high serum vitamin $D$ level would protect individuals from oral diseases $[12,16]$. However, the nonlinearity was not explored in their researches. Thus, the hypothesis of this study was that the relationship between serum vitamin $\mathrm{D}$ and severe periodontitis may be non-linear.

Hence, this study aims to clarify the association of 25 $(\mathrm{OH}) \mathrm{D}$ and severe periodontitis using a typical sample. The data were obtained from the NHANES from 2013 to 2014. The datasets from this period were favorable as they contained information on the latest periodontal examination, as well as severe periodontitis.

\section{Methods}

NHANES protocol approved by NCHS Research Ethics Review Board, and obtained informed consent from all participants (https://www.cdc.gov/nchs/nhanes/ index.htm). The Institutional Review Board at the Shanxi
Provincial People's Hospital determined the analysis used public data sets that did not constitute human subjects research, so human subjects approval was not needed.

\section{Study design and participants}

This is a cross-sectional study. The study was organized based on the list of items for the STROBE (crosssectional studies). Trained staff conducted examinations and interviews through automated data collection where questions were sent directly to the participants or through an agent if necessary. Individuals were enrolled in NHANES data from 2013 to 2014. Inclusion criteria included participants with complete full-mouth periodontal examination (FMPE). Exclusion criteria included: (1) uncompleted with 25-hydroxyvitamin D tests; (2) aged less than 30 years old. A total of 10,175 individuals were screened, out of which 2928 individuals were sampled for the interviews.

\section{Primary variables}

Periodontal examination applied the FMPE protocol, which collects probe measurements from six sites per tooth for all teeth in NHANES. Accordingly, the CDC/ $\mathrm{AAP}$ definitions were based on measures of $\mathrm{AL}$ and $\mathrm{PD}$ at the four interproximal sites per tooth [17] Severe periodontitis was characterized as: $\geq 2$ interproximal sites with attachment loss $(\mathrm{AL}) \geq 6 \mathrm{~mm}$ (not on the same tooth) and $\geq 1$ interproximal site with probing depth (PD) $\geq 5 \mathrm{~mm}$. Mild periodontitis, moderate periodontitis, and no periodontitis definitions were described in Additional file 1: Table S1.

Using the standard protocol, serum 25(OH)D levels were tested in blood samples collected through venipuncture at mobile examination centers (MEC). Serum $25(\mathrm{OH}) \mathrm{D}$ was measured via DiaSorin radioimmunoassay kit (Diasorin, Stillwater, MN, USA) at the National Center for Environmental Health, Center for Disease Control and Prevention. As in previous studies, the serum 25(OH)D levels were classified as normal $(\geq 75 \mathrm{nmol} / \mathrm{ml})$, insufficient $(50-74.9 \mathrm{nmol} / \mathrm{ml})$, deficient $(25-49.9 \mathrm{nmol} / \mathrm{ml})$, and severely deficient $(<25 \mathrm{nmol} / \mathrm{ml})$ [16].

\section{Study variables}

Potential confounding factors were selected from previous studies, including gender, race, body mass index (BMI), age, education, diabetes mellitus, marital status, smoking status, hypertension, poverty-income ratio (PIR), alcohol consumption, hypercholesterolemia, triglyceride, apolipoprotein (B), health insurance, total cholesterol, low-density lipoprotein cholesterol (LDL-c), high-density lipoprotein cholesterol (HDL-c), glycohemoglobin, and fasting blood glucose. The definitions of 
smoking status and alcohol consumption were based on our previous study (Additional file 1) [16]. Patients were considered hypertensive based on a medical report or if they were on antihypertensive drugs. The diagnosis of hypercholesterolemia was based on cholesterol test results or if patients were on lipid-lowering drugs. The race was classified as other race, non-Hispanic white, non-Hispanic black, other Hispanic, or Mexican-American. Health insurance status was captured by the question: "Do you have health insurance or other kinds of health insurance?" As mentioned earlier, the household PIR is obtained by dividing the household income by the poverty criterion. A college degree or above, certain college or associate (AA) degrees, a high school graduation, or below 11th grade are classified based on educational levels. The marital status was categorized into married, separated, never married, living with a partner, divorced, or widowed.

\section{Statistical analysis}

Baseline characteristics were analyzed using means, standard errors (SE), median (min-max), percentages, or frequencies. Continuous variables were compared using analysis of variance (ANOVA) for normally distributed variables, and non-parametric test for not fit the normal distribution. Categorical variables were analyzed using the Chi-square test. We adjusted the $p$ value of multiple groups testing to perform the large number of tests by Bonferroni correction. The effect of $25(\mathrm{OH})$ $\mathrm{D}$ on the severe periodontal disease was examined using multiple logistic regression model. Model I: no adjustment, Model II: adjusted for gender and age, and Model III: adjusted for age, BMI, hypertension, gender, marital status, alcohol consumption, education level, race/Hispanic origin, apolipoprotein (B), hypercholesterolemia, glycohemoglobin, HDL-C, health insurance, total cholesterol, triglyceride, LDL-C, and smoking status. Further, converting classification variables of $25(\mathrm{OH}) \mathrm{D}$ to continuous variables to perform tests for linear trend for robust analysis in the models. To account for $25(\mathrm{OH}) \mathrm{D}$ as a continuous variable, the effect value for severe periodontitis per increase in $25(\mathrm{OH}) \mathrm{D}$ per standard deviation (SD) was determined. a smooth curve fitting and a generalized additive model was established to address the association of severe periodontitis and $25(\mathrm{OH}) \mathrm{D}$. When nonlinearity is detected, the recursive algorithm is first used to calculate the inflection point, and then a two-segment linear regression model is built on both sides of the inflection point. Interaction and stratified analyses were performed based on all variables outlined in Table 1 . Subgroup analysis was performed to assess the potential effect of unmeasured confounding variables. R (http:// www.R-project.org) and Empower-Stats were used for all analyses. Two-sided $p$ values $<0.05$ were considered statistically significant.

\section{Results}

A total of 2928 participants were enrolled in our study (Fig. 1). $10.79 \%(n=316)$ of the participants had severe periodontitis.

Table 1 shows sociodemographic characteristics and other covariates from NHANES (2013-2014). The population mean age was $50 \pm 13.71$ (30-80) years old, with 1425 (48.67\%) males. Glycohemoglobin, apolipoprotein (B), fasting glucose, total cholesterol, LDL-C, triglyceride, and diabetes mellitus were similar in the different groups of serum 25(OH)D. Compared with the deficient, insufficient, and normal groups, individuals with severely deficient $25(\mathrm{OH}) \mathrm{D}$ were likely to have severe periodontal disease, high creatinine, BMI, PIR, alcohol consumption, live single/divorced/widowed, and non-Hispanic black. An opposite pattern was observed for age, HDL-C, education level (college graduate or above), hypercholesterolemia, and current smoking.

The univariate analysis suggested that age, female, race, education level, marital status, PIR, hypertension, glycohemoglobin, health insurance, and smoking status were significantly associated with the severe periodontitis (Additional file 1: Table S2). Also, 25(OH)D were increased in the non-severe periodontitis than severe periodontitis group (Fig. 2).

Table 2 shows serum $25(\mathrm{OH}) \mathrm{D}$ is related to severe periodontitis at three models. The results show that per SD increase in serum $25(\mathrm{OH}) \mathrm{D}$ levels is related to develop severe periodontitis (OR 0.75, 95\% CI 0.63-0.89). An increase in $25(\mathrm{OH}) \mathrm{D}$ levels was associated with a reduced probability of developing severe periodontitis (OR 0.75, 95\% CI 0.63-0.89). In Model I, which was unadjusted, $25(\mathrm{OH}) \mathrm{D}$ was negatively associated with severe periodontitis (OR 0.69, 95\% CI 0.60-0.79). After adjusting for age and gender in Model II, similar results were detected (OR $0.57,95 \%$ CI $0.49-0.66)$. After adjusting significant variables in Additional file 1: Table S2, an increase in 25(OH) $\mathrm{D}$ level was also negatively associated with severe periodontitis (OR $0.75,95 \%$ CI 0.63-0.89). Taken together, these findings indicated that serum 25(OH)D levels were significantly associated with reduced risk of severe periodontal disease. Therefore, we also established a generalized additive model and a smooth curve fitting to assess the relation between severe periodontitis and $25(\mathrm{OH}) \mathrm{D}$ (Fig 3). The result indicates that the two piecewise linear regression was more suitable for fitting the association between $25(\mathrm{OH}) \mathrm{D}$ and severe periodontitis because it can accurately represent the relationship between severe periodontitis and $25(\mathrm{OH}) \mathrm{D}$. We calculated the inflection point was $102(\mathrm{nmol} / \mathrm{L})$ through the two-piecewise linear 
Table 1 Baseline characteristics of the participates

\begin{tabular}{|c|c|c|c|c|}
\hline \multirow[t]{2}{*}{ Characteristics } & \multicolumn{4}{|c|}{ Serum 25-hydroxyvitamin $\mathrm{D}, \mathrm{nmol} / \mathrm{L}$} \\
\hline & Severely deficient $(<25)$ & Deficient (25-49.9) & Insufficient (50-74.9) & Normal $(\geq 75)$ \\
\hline N & 105 & 697 & 1104 & 929 \\
\hline Age(year) & $46.03 \pm 11.88$ & $46.20 \pm 11.95$ & $48.48 \pm 12.81$ & $55.14 \pm 14.61$ \\
\hline \multicolumn{5}{|l|}{ Gender } \\
\hline Male & $46(43.81 \%)$ & $352(50.50 \%)$ & $584(52.90 \%)$ & 389 (41.87\%) \\
\hline Female & $59(56.19 \%)$ & 345 (49.50\%) & 520 (47.10\%) & $540(58.13 \%)$ \\
\hline Creatinine, urine(umol/L) & $10,961(6806,16,663)$ & $10,210(5569,1547)$ & 9370. $(5304,14,409)$ & $7602(4243,12,287)$ \\
\hline Apolipoprotein (B) (mg/dL) & $90.44 \pm 20.80$ & $93.97 \pm 24.13$ & $94.20 \pm 26.18$ & $92.33 \pm 25.44$ \\
\hline $\mathrm{BMI}(\mathrm{Kg} / \mathrm{m} 2)$ & $33.19 \pm 8.83$ & $30.87 \pm 7.61$ & $29.07 \pm 6.18$ & $27.96 \pm 6.51$ \\
\hline Glycohemoglobin (\%) & $5.89 \pm 1.26$ & $5.83 \pm 1.21$ & $5.70 \pm 0.94$ & $5.70 \pm 0.96$ \\
\hline Fasting Glucose (mmol/L) & $6.00 \pm 2.36$ & $6.10 \pm 2.11$ & $5.97 \pm 1.80$ & $5.83 \pm 1.49$ \\
\hline Direct HDL-Cholesterol (mmol/L) & $1.34 \pm 0.39$ & $1.30 \pm 0.39$ & $1.33 \pm 0.39$ & $1.50 \pm 0.46$ \\
\hline Total Cholesterol (mmol/L) & $4.85 \pm 0.90$ & $5.03 \pm 1.13$ & $5.02 \pm 1.00$ & $5.06 \pm 1.18$ \\
\hline Triglyceride (mmol/L) & $1.01(0.71,1.42)$ & $1.03(0.72,1.67)$ & $1.17(0.78-1.82)$ & $1.06(0.74,1.53)$ \\
\hline LDL-cholesterol (mmol/L) & $2.85 \pm 0.73$ & $3.00 \pm 0.87$ & $3.00 \pm 0.92$ & $2.98 \pm 0.91$ \\
\hline \multicolumn{5}{|l|}{ Race/Hispanic origin } \\
\hline Mexican American & $12(11.43 \%)$ & $144(20.66 \%)$ & $184(16.67 \%)$ & 75 (8.07\%) \\
\hline Other Hispanic & $6(5.71 \%)$ & 59 (8.46\%) & $126(11.41 \%)$ & $51(5.49 \%)$ \\
\hline Non-Hispanic White & $14(13.33 \%)$ & $172(24.68 \%)$ & $467(42.30 \%)$ & $589(63.40 \%)$ \\
\hline Non-Hispanic Black & $59(56.19 \%)$ & $198(28.41 \%)$ & 147 (13.32\%) & $95(10.23 \%)$ \\
\hline Other Race & $14(13.33 \%)$ & $124(17.79 \%)$ & $180(16.30 \%)$ & $119(12.81 \%)$ \\
\hline \multicolumn{5}{|l|}{ Education level } \\
\hline Less than 11 th grade & $19(18.10 \%)$ & $159(22.81 \%)$ & $221(20.02 \%)$ & 117 (12.61\%) \\
\hline High school graduate/GED & $28(26.67 \%)$ & $154(22.09 \%)$ & $224(20.29 \%)$ & $170(18.32 \%)$ \\
\hline Some college or AA degree & $36(34.29 \%)$ & 205 (29.41\%) & $316(28.62 \%)$ & 290 (31.25\%) \\
\hline College graduate or above & $22(20.95 \%)$ & $179(25.68 \%)$ & $343(31.07 \%)$ & 351 (37.82\%) \\
\hline \multicolumn{5}{|l|}{ Marital status } \\
\hline Single/divorced/widowed & $31(29.52 \%)$ & $142(20.37 \%)$ & $216(19.58 \%)$ & 205 (22.07\%) \\
\hline Never married & $26(24.76 \%)$ & $115(16.50 \%)$ & $96(8.70 \%)$ & $85(9.15 \%)$ \\
\hline Married/living as married & $48(45.71 \%)$ & $440(63.13 \%)$ & 791 (71.71\%) & 639 (68.78\%) \\
\hline \multicolumn{5}{|l|}{ Ratio of family income to poverty } \\
\hline$<1.3$ & $41(40.20 \%)$ & 231 (36.09\%) & $270(26.63 \%)$ & 185 (21.54\%) \\
\hline $1.3-3.5$ & $44(43.14 \%)$ & $220(34.38 \%)$ & $357(35.21 \%)$ & $253(29.45 \%)$ \\
\hline$>3.5$ & 17 (16.67\%) & $189(29.53 \%)$ & $387(38.17 \%)$ & 421 (49.01\%) \\
\hline \multicolumn{5}{|l|}{ Alcohol consumption } \\
\hline No & $21(21.00 \%)$ & $188(29.15 \%)$ & $242(23.16 \%)$ & $226(25.54 \%)$ \\
\hline Yes & 79 (79.00\%) & 457 (70.85\%) & 803 (76.84\%) & 659 (74.46\%) \\
\hline \multicolumn{5}{|l|}{ Hypertension } \\
\hline No & $62(59.05 \%)$ & 459 (65.85\%) & 777 (70.38\%) & $535(57.65 \%)$ \\
\hline Yes & 43 (40.95\%) & $238(34.15 \%)$ & $327(29.62 \%)$ & $393(42.35 \%)$ \\
\hline \multicolumn{5}{|l|}{ Hypercholesterolemia } \\
\hline No & $74(70.48 \%)$ & $484(69.44 \%)$ & $693(62.77 \%)$ & $503(54.14 \%)$ \\
\hline Yes & $31(29.52 \%)$ & $207(29.70 \%)$ & 404 (36.59\%) & 425 (45.75\%) \\
\hline \multicolumn{5}{|l|}{ Diabetes mellitus } \\
\hline No & $91(86.67 \%)$ & $625(89.80 \%)$ & $983(89.04 \%)$ & $812(87.50 \%)$ \\
\hline Yes & $14(13.33 \%)$ & $71(10.20 \%)$ & $121(10.96 \%)$ & $116(12.50 \%)$ \\
\hline \multicolumn{5}{|l|}{ Health insurance } \\
\hline No & $28(26.67 \%)$ & 204 (29.27\%) & 242 (21.94\%) & 106 (11.41\%) \\
\hline Yes & 77 (73.33\%) & 493 (70.73\%) & 861 (78.06\%) & $823(88.59 \%)$ \\
\hline Smoking status & & & & \\
\hline
\end{tabular}


Table 1 (continued)

\begin{tabular}{lllll}
\hline Characteristics & \multicolumn{4}{l}{ Serum 25-hydroxyvitamin D, nmol/L } \\
\cline { 2 - 5 } & Severely deficient (<25) & Deficient (25-49.9) & Insufficient (50-74.9) & Normal $(\geq 75)$ \\
\hline Never smoking & $54(51.43 \%)$ & $406(58.33 \%)$ & $646(58.51 \%)$ & $562(60.56 \%)$ \\
Former smoking & $34(32.38 \%)$ & $135(19.40 \%)$ & $147(13.32 \%)$ & $100(10.78 \%)$ \\
Current smoking & $17(16.19 \%)$ & $155(22.27 \%)$ & $311(28.17 \%)$ & $266(28.66 \%)$ \\
Severe periodontitis & & & \\
No & $81(77.14 \%)$ & $596(85.51 \%)$ & $997(90.31 \%)$ & $860(92.57 \%)$ \\
Yes & $24(22.86 \%)$ & $101(14.49 \%)$ & $107(9.69 \%)$ & $69(7.43 \%)$ \\
\hline
\end{tabular}

Bold denotes statistical signifcance at $\mathrm{p}<0.05$

$p$ values were calculated using the Chi-square, analysis of variance (ANOVA) and Bonferroni correction

BMI, body mass index; AA, associate degree; GED, General Educational Development; HDL-Cholesterol, high-density lipoprotein cholesterol; LDL-Cholesterol, lowdensity lipoprotein cholesterol

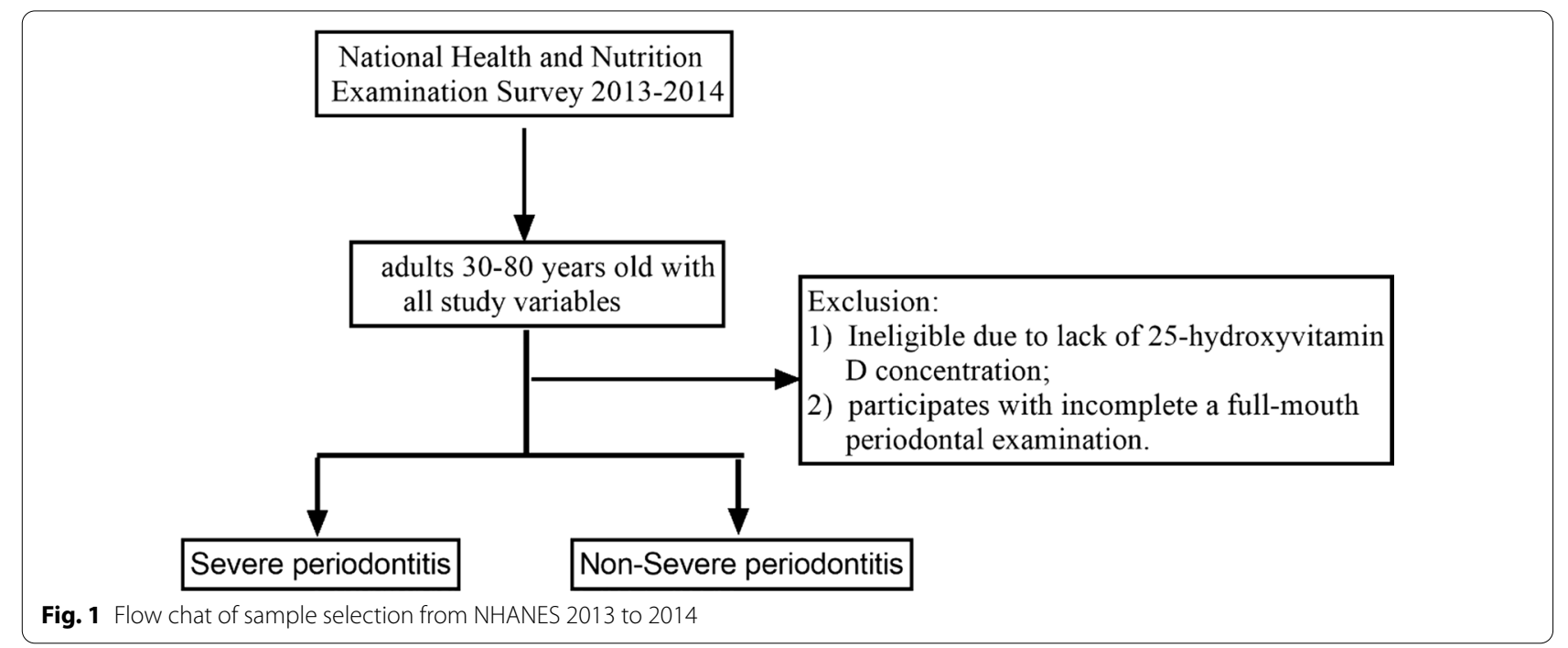

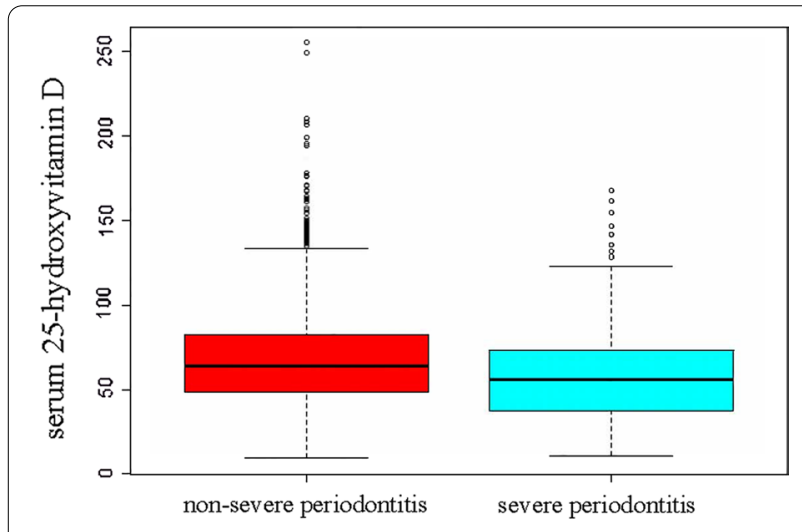

Fig. 2 Comparison of serum 25-hydroxyvitamin D value between non-severe periodontitis and severe periodontitis group by non-parametric test $(F=27.9, p<0.05)$. The bottom and top edges of each box represent the first and third quartiles, respectively, the band within the box represents the median value regression and recursive algorithm. On the left side of the inflection point $(25(\mathrm{OH}) \mathrm{D} \leq 102 \mathrm{nmol} / \mathrm{L})$, the effect size was 0.98 and $95 \% \mathrm{CI}$ was $0.98-0.99(25(\mathrm{OH}) \mathrm{D}$ per $1 \mathrm{nmol} / \mathrm{L}$ increments). On the right side of the inflection point $(25(\mathrm{OH}) \mathrm{D}>102 \mathrm{nmol} / \mathrm{L})$, the effect size was 0.99 and $95 \% \mathrm{CI}$ was $0.98-1.01$ (Table 3).

Subgroup analysis results are shown in Additional file 1: Table S3. Race, alcohol consumption, diabetes mellitus, and health insurance were potential confounders of $25(\mathrm{OH}) \mathrm{D}$ and severe periodontitis $(P<0.05)$.

\section{Discussion}

This study is one of the largest epidemiologic studies to investigate the association between serum $25(\mathrm{OH}) \mathrm{D}$ and severe periodontitis. After adjusting for confounders in the full adjusted model, we found that $25(\mathrm{OH}) \mathrm{D}$ is negatively associated with severe periodontitis. The increase 
Table 2 Multiple logistic regression model for the association between 25(OH)D (nmol/L) and severe periodontitis in different models

\begin{tabular}{|c|c|c|c|c|c|c|}
\hline \multirow[t]{2}{*}{ Exposure } & \multicolumn{2}{|l|}{ Model I } & \multicolumn{2}{|l|}{ Model II } & \multicolumn{2}{|l|}{ Model III } \\
\hline & OR, $95 \% \mathrm{Cl}$ & $p$ value & OR, $95 \% \mathrm{Cl}$ & $p$ value & $\mathrm{OR}, 95 \% \mathrm{Cl}$ & $p$ value \\
\hline \multicolumn{7}{|l|}{ Serum 25(OH)D, nmol/L } \\
\hline Severely deficient $(<25)$ & Reference & & Reference & & Reference & \\
\hline Deficient (25-49.9) & $0.57(0.35,0.94)$ & 0.029 & $0.51(0.30,0.86)$ & 0.011 & $0.68(0.37,1.24)$ & 0.208 \\
\hline Insufficient (50-74.9) & $0.36(0.22,0.60)$ & $<0.001$ & $0.27(0.16,0.45)$ & $<0.001$ & $0.58(0.31,1.08)$ & 0.086 \\
\hline Normal $(\geq 75)$ & $0.27(0.16,0.45)$ & $<0.001$ & $0.16(0.09,0.28)$ & $<0.001$ & $0.36(0.19,0.69)$ & 0.002 \\
\hline P for trend & $<0.001$ & & & $<0.001$ & & \\
\hline Serum 25(OH)D, per SD & $0.69(0.60,0.79)$ & $<0.001$ & $0.57(0.49,0.66)$ & $<0.001$ & $0.75(0.63,0.89)$ & 0.001 \\
\hline
\end{tabular}

Model I: adjust for none

Model II: adjust for gender, age

Model III: adjust for gender; hypertension; direct HDL-Cholesterol, age, race/Hispanic origin, glycohemoglobin, education level, diabetes mellitus, BMI, marital status, creatinine, health insurance, smoking status, ratio of family income to poverty

Table 3 The results of the two-piecewise linear model

\begin{tabular}{lll}
\hline & Severe periodontitis (OR, 95\%Cl) & $\boldsymbol{p}$ value \\
\hline Fitting model by standard linear regression, per SD increments & $0.75(0.64,0.89)$ & 0.001 \\
Fitting model by two-piecewise linear regression & & 102 \\
Inflection point of lactate dehydrogenase, $\mathrm{nmol} / \mathrm{L}$ & $0.98(0.98,0.99)$ \\
$\leq 102$ & $0.99(0.98,1.01)$ & 0.001 \\
$>102$ & 0.798 \\
\hline
\end{tabular}

Adjusted: gender; hypertension; HDL-Cholesterol, age, race/Hispanic origin, glycohemoglobin, education level, diabetes mellitus, BMI, marital status, creatinine, health insurance, smoking status, ratio of family income to poverty

BMI, body mass index; HDL, high-density lipoprotein; SD, standard deviation

in per SD serum $25(\mathrm{OH}) \mathrm{D}$ levels corresponded to a $25 \%$ decrease in the probability of severe periodontitis. Besides, a non-linear relationship was detected between severe periodontitis and serum $25(\mathrm{OH}) \mathrm{D}$. On the left side of the inflection point, the risk of perodontitis in U.S. participants was reduced by $2 \%$ for each additional $1 \mathrm{nmol} / \mathrm{L}$ of serum $25(\mathrm{OH}) \mathrm{D}$. On the right side of the inflection point, the relationship cannot be observed (95\% CI was $0.99,0.98-1.01, P=0.798)$. Besides, there was a stronger association between $25(\mathrm{OH}) \mathrm{D}$ and severe periodontitis in non-Hispanic white, alcohol consumption, diabetes, and health insurance populations after subgroup analysis.

Previous studies showed that the serum 25(OH) D level or Vitamin D intake were related to periodontitis [12, 18, 19]. However, some studies reported no disparity between $25(\mathrm{OH}) \mathrm{D}$ and periodontal disease severity $[5,20]$. A cohort study using NHANES III data found $25(\mathrm{OH}) \mathrm{D}$ is not associated with periodontitis in matched populations younger than 50 years old [21]. Besides, NHANES III data were limited to sites without any attachment loss due to periodontitis. Recent studies indicated that the level of vitamin D intake is related to periodontitis using recently NHANES data (2013-2014)
[14]. NHANES data (2013-2014) and the periodontal examination was based on FMPE protocol, which was more accurate than before. Our study is similar with previous studies that serum $25(\mathrm{OH}) \mathrm{D}$ were also related with severe periodontitis. Recently, a series of studies reported that serum $25(\mathrm{OH}) \mathrm{D}$ was associated with periodontitis $[2,12,22]$. However, nonlinearity and subgroup analysis were not performed in their researches. Therefore, the contribution of this study was the discovery of a nonlinear and threshold effect on the relationship between $25(\mathrm{OH}) \mathrm{D}$ and severe periodontitis. The immune and inflammatory response against periodontal pathogens are suggested to be triggered by the host immune system [23]. Moreover, some results have shown that during severe periodontitis, vitamin D receptors in the immune system cells protect periodontal tissue endothelium and reduce the release of $B$ and $T$ lymphocytes caused by periodontal pathogens [24].

Subgroup analysis was used to assess the independent relationship between $25(\mathrm{OH}) \mathrm{D}$ and severe periodontitis. Gender, age, race, alcohol consumption, education level, apolipoprotein, BMI, marital status, hypertension, PIR, diabetes, glycohemoglobin, fasting glucose, 
health insurance, smoking status, total cholesterol, triglyceride, and LDL-C were used as category variables, of which interactions were detected in non-Hispanic white, alcohol consumption, diabetes, and health insurance populations. The reason may explain as people with health insurance pay more attention to oral health. Also, study indicated that diabetes increases the risk of periodontal diseases, also, inflammatory diseases may increase insulin resistance [25]. Moreover, we found the level of serum 25(OH)D in non-Hispanic white people was higher than that in other people, and severe periodontitis was lower than that in other races, which were consistent with previous study [26]. A cross-sectional study found no dependence between alcohol and severe periodontal disease [27]. While alcohol consumption is associated with vitamin D [28], which may affect the periodontitis, similar to our results. Therefore, in American adults, there is an urgent need to prevent alcohol consumption at low levels of serum $25(\mathrm{OH}) \mathrm{D}$ with severe periodontitis.

The present study is of clinical value. To the best of our knowledge, we use the latest data from NHANES to assess the association of serum 25(OH)D levels and periodontitis. The results of this study may be useful for further pathogenesis research. Our research will also contribute to the evidence to the literature.

Our study has some advantages. (1) We address the nonlinear problem in our current study and explore it further. (2) Due to a cross-sectional study; hence, if may be prone to biases. Because we used strict statistical adjustments, the remaining confounding factors were minimized. (3) We convert the target-independent variable into both a categorical and a continuous variable. This method may restrict data analyzed and enhance the robustness of the results. (4) Effect correction factor analysis enabled this study to make better use of the data to produce stable conduction in different subgroups.

This research also has some shortcomings. First, our findings are based on a U.S. population, therefore, these findings may not be extrapolated to other nationalities. Second, some important confounders such as the sunshine time and daily consumption that affect the levels of vitamin $\mathrm{D}$ and the inflammatory status were not achieved. Nevertheless, our results indicated $25(\mathrm{OH})$ $\mathrm{D}$ was negatively related to severe periodontal disease. Taking vitamin D or receiving more sunshine may cause the relationship of the smooth curve fitting in Fig. 3 to disappear. Third, due to the definition of non-severe periodontitis include mild and moderate periodontitis patients, which may lead to some selected bias. Forth, cross-sectional studies alone cannot establish a causal connection between severe periodontitis and 25(OH)D. Therefore, well-designed investigates should be explored

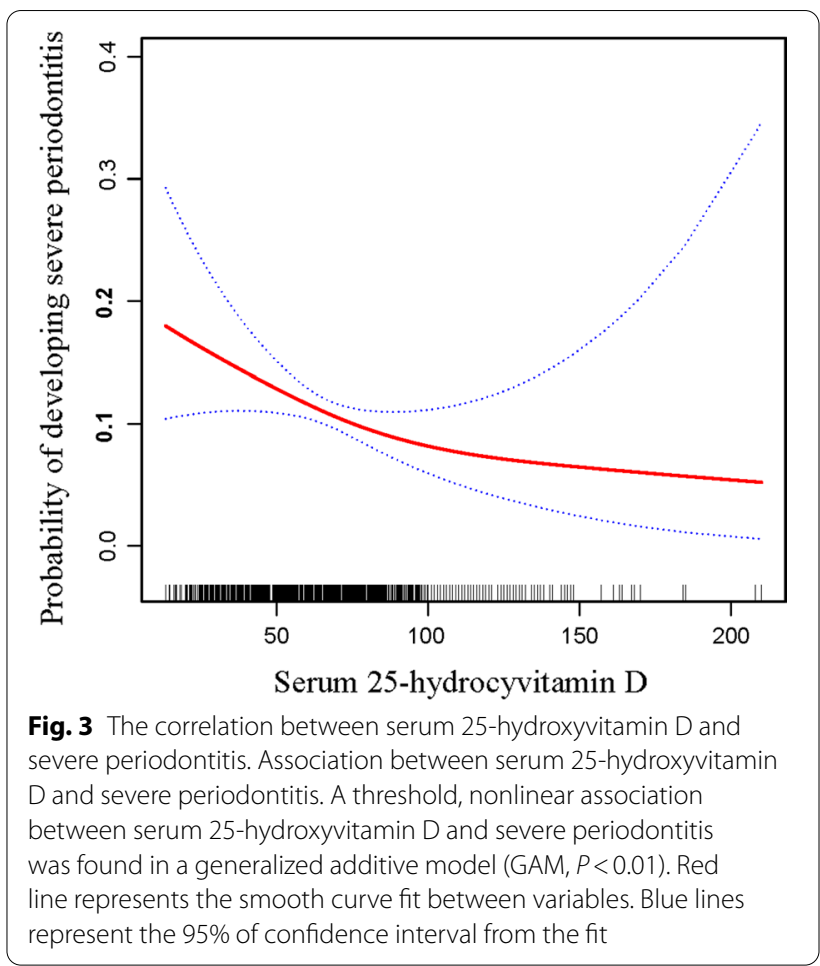

to determine the potential anti-inflammatory effect of $25(\mathrm{OH}) \mathrm{D}$ on periodontitis.

\section{Conclusion}

The relationship between serum 25(OH)D and severe periodontitis in our study population is non-linear. Serum 25(OH)Dwas negatively related to severe periodontitis when it is less $102 \mathrm{nmol} / \mathrm{L}$. The association, however, should be further evaluated using long-term cohort studies.

\section{Abbreviations}

NHANES: National Health and Nutrition Examination Survey; 25(OH)D: 25-Hydroxyvitamin D; NCHS: National Center for Health Statistics; FMPE: Fullmouth periodontal examination; BMI: Body mass index; AA: Associate degree; GED: General Educational Development; HDL-Cholesterol: High-density lipoprotein cholesterol; LDL-Cholesterol: Low-density lipoprotein cholesterol.

\section{Supplementary Information}

The online version contains supplementary material available at https://doi. org/10.1186/s12903-021-01850-3.

Additional file 1: Table S1. Case definitions proposed for population based surveillance to periodontitis; Table S2. Univariate analysis for severe periodontitisUnivariate analysis for severe periodontitis; Table S3. Subgroup analysis for the interaction of $25(\mathrm{OH}) \mathrm{D}$ levels in different group subgroup analysis for the interaction of $25(\mathrm{OH}) \mathrm{D}$ levels in different group. 


\section{Acknowledgements \\ Not applicable}

\section{Authors' contributions}

FZ wrote the manuscript and collected the participates information. YZ performed statistical analysis. XH, XW draft the table and NM, RS draft the figure. JS analyzed and interpreted the patients' general indices. All authors read and approved the final manuscript.

\section{Funding}

This manuscript was supported by the project from Health Commission of Shanxi Province (No. 2020TD17).

\section{Availability of data and materials}

The NHANES data of this study are openly available at https://www.cdc.gov/ nchs/nhanes/default.aspx.

\section{Declarations}

Ethics approval and consent to participate

NHANES protocol approved by NCHS Research Ethics Review Board, and obtained informed consent from all participants.

\section{Consent for publication}

Not applicable.

\section{Competing interests}

The authors declare that they have no competing interests.

\section{Author details}

${ }^{1}$ Shanxi Medical University School and Hospital of Stomatology, Taiyuan 030012, Shanxi Province, China. ${ }^{2}$ Department of Emergency Medicine, The Second Xiangya Hospital of Central South University, 139 Renmin road, Changsha 410011, Hunan Province, China. ${ }^{3}$ Department of Oral Medicine, The Fifth Clinical Medical College of Shanxi Medical University, 22 Shuangtasi Street, Taiyuan 030012, Shanxi Province, China.

Received: 9 June 2021 Accepted: 21 September 2021 Published online: 27 September 2021

\section{References}

1. Kassebaum NJ, Bernabe E, Dahiya M, Bhandari B, Murray CJ, Marcenes W. Global burden of severe periodontitis in 1990-2010: a systematic review and meta-regression. J Dent Res. 2014;93(11):1045-53.

2. Eke PI, Thornton-Evans GO, Wei L, Borgnakke WS, Dye BA, Genco RJ. Periodontitis in US Adults: National Health and Nutrition Examination Survey 2009-2014. J Am Dental Assoc (1939) 2018, 149(7):576-588 e576.

3. Otomo-Corgel J, Pucher JJ, Rethman MP, Reynolds MA. State of the science: chronic periodontitis and systemic health. J Evid Based Dent Pract. 2012;12(3 Suppl):20-8.

4. Buset SL, Walter C, Friedmann A, Weiger R, Borgnakke WS, Zitzmann NU. Are periodontal diseases really silent? A systematic review of their effect on quality of life. J Clin Periodontol. 2016;43(4):333-44.

5. Botelho J, Machado V, Proenca L, Delgado AS, Mendes JJ. Vitamin $D$ deficiency and oral health: a comprehensive review. Nutrients. 2020;12(5):1471.

6. Alqaderi H, Goodson JM, Agaku I. Association between sleep and severe periodontitis in a nationally representative adult US population. J Periodontol. 2020;91(6):767-74

7. Lee WC, Fu E, Li CH, Huang RY, Chiu HC, Cheng WC, Chen WL. Association between periodontitis and pulmonary function based on the Third National Health and Nutrition Examination Survey (NHANES III). J Clin Periodontol. 2020;47(7):788-95.

8. Pihlstrom BL, Michalowicz BS, Johnson NW. Periodontal diseases. Lancet. 2005;366(9499):1809-20.
9. Aguiar M, Andronis L, Pallan M, Hogler W, Frew E. The economic case for prevention of population vitamin D deficiency: a modelling study using data from England and Wales. Eur J Clin Nutr. 2020;74(5):825-33.

10. Fathi N, Ahmadian E, Shahi S, Roshangar L, Khan H, Kouhsoltani M, Maleki Dizaj S, Sharifi S. Role of vitamin D and vitamin D receptor (VDR) in oral cancer. Biomed Pharmacother Biomedecine pharmacotherapie. 2019;109:391-401.

11. Dragonas P, El-Sioufi I, Bobetsis YA, Madianos PN. Association of vitamin D with periodontal disease: a narrative review. Oral Health Prev Dent. 2020;18(2):103-14.

12. Isola G, Alibrandi A, Rapisarda E, Matarese G, Williams RC, Leonardi R. Association of vitamin $D$ in patients with periodontitis: A cross-sectional study. J Periodontal Res. 2020;55:602-12.

13. Slots J. Periodontitis: facts, fallacies and the future. Periodontol 2000. 2017;75(1):7-23.

14. Luo PP, Xu HS, Chen YW, Wu SP. Periodontal disease severity is associated with micronutrient intake. Aust Dent J. 2018;63(2):193-201.

15. D'Andrea A, Chang C, Phillips JH, Lanier LL. Regulation of T cell lymphokine production by killer cell inhibitory receptor recognition of self HLA class I alleles. J Exp Med. 1996;184(2):789-94.

16. Zhou F, Zhou Y, Shi J. The association between serum 25-hydroxyvitamin D levels and dental caries in US adults. Oral Dis. 2020;26:1537-47.

17. Eke PI, Page RC, Wei L, Thornton-Evans G, Genco RJ. Update of the case definitions for population-based surveillance of periodontitis. J Periodontol. 2012:83(12):1449-54.

18. Anbarcioglu E, Kirtiloglu T, Ozturk A, Kolbakir F, Acikgoz G, Colak R. Vitamin D deficiency in patients with aggressive periodontitis. Oral Dis. 2019;25(1):242-9.

19. Agrawal AA, Kolte AP, Kolte RA, Chari S, Gupta M, Pakhmode R. Evaluation and comparison of serum vitamin D and calcium levels in periodontally healthy, chronic gingivitis and chronic periodontitis in patients with and without diabetes mellitus - a cross-sectional study. Acta Odontol Scand. 2019;77(8):592-9.

20. Costantini E, Sinjari B, Piscopo F, Porreca A, Reale M, Caputi S, Murmura G. Evaluation of salivary cytokines and vitamin D levels in periodontopathic patients. Int J Mol Sci. 2020;21(8):2669.

21. Dietrich T, Nunn M, Dawson-Hughes B, Bischoff-Ferrari HA. Association between serum concentrations of 25-hydroxyvitamin $D$ and gingival inflammation. Am J Clin Nutr. 2005;82(3):575-80

22. Millen AE, Hovey KM, LaMonte MJ, Swanson M, Andrews CA, Kluczynski MA, Genco RJ, Wactawski-Wende J. Plasma 25-hydroxyvitamin D concentrations and periodontal disease in postmenopausal women. J Periodontol. 2013;84(9):1243-56.

23. Hildebolt CF. Effect of vitamin D and calcium on periodontitis. J Periodontol. 2005;76(9):1576-87.

24. Lai H, Detrick B, Fishman EK, Gerstenblith G, Brinker JA, Hollis BW, Bartlett $\mathrm{J}$, Cofrancesco J Jr, Tong W, Tai H, et al. Vitamin D deficiency is associated with the development of subclinical coronary artery disease in African Americans with HIV infection: a preliminary study. J Investig Med. 2012;60(5):801-7

25. Mealey BL, Oates TW. American Academy of P: Diabetes mellitus and periodontal diseases. J Periodontol. 2006;77(8):1289-303.

26. Eke PI, Dye BA, Wei L, Slade GD, Thornton-Evans GO, Borgnakke WS, Taylor GW, Page RC, Beck JD, Genco RJ. Update on prevalence of periodontitis in adults in the United States: NHANES 2009 to 2012. J Periodontol. 2015;86(5):611-22

27. Pinto-Filho JM, Ribeiro LSF, Sartori L, Dos Santos JN, Ramalho LMP, Cury PR. Association between alcohol dependence and both periodontal disease and tooth loss: a cross-sectional study. Environ Sci Pollut Res Int. 2018:25(29):29089-95.

28. Tardelli VS, Lago M, Silveira DXD, Fidalgo TM. Vitamin D and alcohol: a review of the current literature. Psychiatry Res. 2017:248:83-6.

\section{Publisher's Note}

Springer Nature remains neutral with regard to jurisdictional claims in published maps and institutional affiliations. 